\title{
OPEN SPATIAL DATA FOR DECISION MAKING - REQUIREMENTS AND QUALITATIVE CONSIDERATIONS
}

\author{
Hardy Pundt \\ Harz University of Applied Sciences \\ Friedrichstr. 57-59, 38855 Wernigerode, Germany
}

\begin{abstract}
Open data play an increasing role in various societal fields, administration, economy, science and others. This concerns also open, spatial data which can be used in space-related decision-making. Open spatial data will be introduced as an important source of information. However, open spatial data must meet specific qualitative requirements. Pros and Cons will be identified and recommendations will be given which characteristics should be considered when using such data. The experiences described are based on the results of a transdisciplinary project that was aimed at deciding on prioritized climate change adaptation measures.
\end{abstract}

\section{KEYWORDS}

Spatial Decision Support Systems, Open Data, Geographical Information Systems, Web Mapping, Geodata

\section{INTRODUCTION}

"Data is a key resource in the modern world" (Kitchin 2014). Open data, however, has raised interest during the last decade in various sectors, administrations, as well as enterprises. Especially in society, open data play an important role because they can be accessed and used by citizens in a manner that is only possible through the wide spread of Web- and mobile applications. Open data has various advantages that makes them an adequate resource to support computer-aided data analysis and decision-making. Open spatial data, as a specific type of open data, can be used widely in different sectors, such as science, governmental administration, business, and by citizens in view of the saying that more than $80 \%$ of decisions made in economy and administration rely, at least to a certain extent, on spatial information.

Benefits of using open data concern, among others, (no) costs and large reuse potential, availability and accessibility, whereas risks are seen concerning privacy and fraud, inaccuracy and legality. But there are more constraints and considerations that have to be taken into account when evaluating the benefits of open, spatial data. Furthermore, the usage of such data should be seen from different perspectives, because the requirements in business, government, science or concerning the usage by citizens are diverse.

This paper will introduce briefly what open, spatial data means in terms of different data types, e. g. qualitative vs. quantitative data, structured, semi-structured and unstructured data, captured, exhaust, transient and derived data, and others, as Kitchin (2014) proposes such categories. The paper will summarize some benefits, as well as risks of applying open data. It will describe an example that explains difficulties and opportunities of developing a Web-based, online mapping system that includes, among others, open spatial data. The example is thought to demonstrate that openness can lead to more effective decision making, if specific quality constraints are considered. This will lead to conclusions that affect the ongoing discussion on proper usage of open data to support spatial analysis and decision making. 


\section{OPEN, SPATIAL DATA}

Open data are seen commonly as data that are published on the Internet in a way that anyone can freely use, re-use and redistribute this data (Open Knowledge Foundation 2021). Open government data is data that is owned by a public sector body (Kucera \& Chlapek, 2014), whereas open data in business means often each kind of data that is abundant and free which makes it a potential source of great opportunities for all businesses, regardless of their location, size or sector (NibusinessInfo 2021). Open, spatial data include "volunteered geographic information (VGI)" a term that was introduced by Goodchild (2007).

Van Loenen et al. (2018) have emphasized that, "in a rather naïve way, it was thought that open data could be used directly by everyone, by all citizens and businesses" but they conclude that this is not the case. This citation confirms experiences that were gathered during a project ("BebeR") that was based on a living lab approach thus including actors from administration, organizations, education and citizens (Pundt and Heilmann 2020). It will be described later in more detail. The steps to establish such a "common data platform" to serve all "concerned parties" raised some challenges that were not expected at the beginning of the project. At the end, however, it was discovered that specific problems that occurred were due to lacking "openness" of data. Discussions of the problems led to the result that open data require the proper consideration of qualitative, security and other constraints, otherwise the acceptance of potential data users is low. Reliability of open data, however, becomes a central quality constraint at that point. Reliability concerns different components, such as the data collection procedures, quality management, data security and others.

In general, open, spatial data can be provided in two ways, by default and by request. Figure 1 is presenting the approaches. Whereas in the first case data are collected and provided without considering who possibly will use the data, the second case describes a situation in which a user asks for the provision of specific data for particular purposes. In such a case, data will only be provided if a formally correct request has been sent.

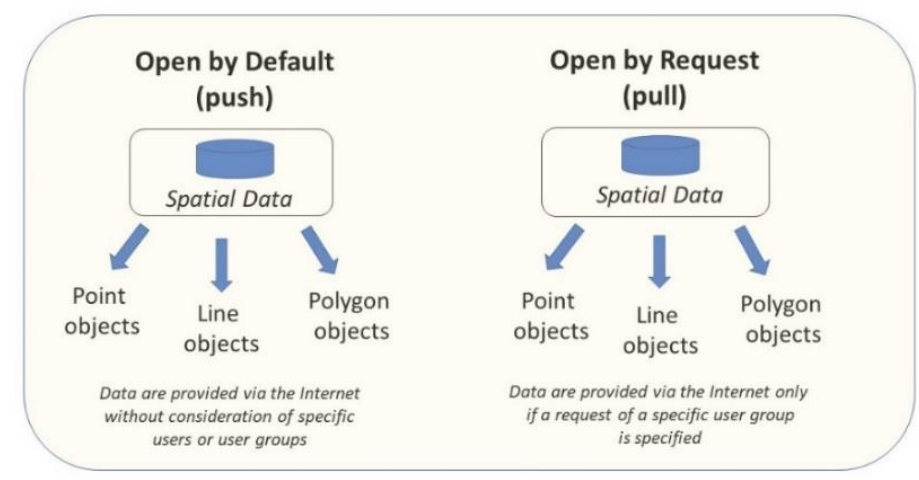

Figure 1. Open data by default and by request (Hörster and Kärle (2019), modified). Adopted concerning a focus on open, spatial data, thus representing point-, line- or polygon objects

Klessmann et al (2012) summarized some pros and cons of open data. Table 1 presents an extended version, including aspects suggested by the Open Knowledge Foundation (OKF 2021), Jackson et al. (2013) and own experiences related to the BebeR-project.

Table 1. Pros and cons of open spatial data: experiences from Klessmann et al (2012), Jackson et al. (2013) and the BebeR-project

\begin{tabular}{|c|c|}
\hline Pros & Cons \\
\hline Klessmann et al. 2012 : & Klessmann et al.2012: \\
\hline Re-use and access & Assessment of quality is difficult \\
\hline Achieving greater audience & Metadata often lacking \\
\hline Enhances overall information basis & $\begin{array}{l}\text { Context-knowledge often not available, which raises the } \\
\text { danger of data misuse }\end{array}$ \\
\hline Jackson et al.2013: & \\
\hline $\begin{array}{l}\text { Open spatial data and VGI means to bring the general } \\
\text { public into the realm of map production functions } \\
\text { traditionally reserved for official agencies }\end{array}$ & $\begin{array}{l}\text { Jackson et al.2013: } \\
\text { Spatial errors between different open datasets } \\
\text { Differences in completeness }\end{array}$ \\
\hline
\end{tabular}


Open data and VGI can extend helpfully official, administrative datasets

BebeR (Pundt 2018; Pundt and Heilmann 2020): Supporting integration of different datasets (data sharing and redistribution)

Enabling integration of spatial layers that would possibly be disregarded

Supporting multi-perspective view on problems and problem solutions

Improving of decision making, possibly accomplish information benefits due to adding open data

Possibly more current data compared with official datasets

Easing data acquisition due to voluntary citizens that act as data collectors or "sensors"
Volunteered data is usually provided with little to no information on mapping standards, quality control procedures, and metadata in general

BebeR (Pundt 2018; Pundt and Heilmann 2020):

Reliability of data in terms of quality parameters and security is difficult, if not impossible, to evaluate (e. g. completeness, spatial accuracy, currentness of data) Insufficient spatial accuracy

Improper spatial reference systems or scales

Unsureness concerning professional data collection procedures

\section{INTEGRATION OF OPEN DATA IN AN ONLINE MAPPING SYSTEM}

\subsection{Project Background}

From 2017 - 2020, the "BebeR-project" has been carried out. The project was aimed at developing and assessing the vulnerability of mountainous areas concerning soil erosion taking into account the challenges provoked by climate change. The investigation area for the development of vulnerability assessment methods was the administrative district of Mansfeld-Südharz, situated in the south of the German federal state of Saxony-Anhalt. Within the framework of carrying out computer simulations it appeared that heavy rainfall events in the concerned region might increase in future. Heavy rainfall, however, can lead to cumulative soil erosion which can, in turn, cause damages in terms of soil loss, as well as damages to infrastructures such as roads, bridges, or houses. These possible future events require decision making to mitigate the threats. This cannot be done without considering the different interests in the region: water management, nature protection, agriculture and forestry, regional planning, settling, urbanization and economy. Due to this situation, various partners were collaborating in the BebeR-project to discuss related problems and alternative solutions, taking into account possibly occurring and varying climate change scenarios (Pundt 2018).

\subsection{Integration of Different Kinds of Spatial Data in an Online Mapping System}

Spatial decision making in multi-actor projects must necessarily be based on comprehensive and adequate data. Within the BebeR-project, establishing such a comprehensive spatial database was an important step to enable the participating stakeholders, including scientists, different administrational and further partners, to use the data from not only one, but all concerned sectors. The intention is, that looking across sectoral borders may lead to more comprehensive and qualitatively better decisions instead of wearing sectoral blinkers. The database was conceptualized and implemented during a foregoing project, and further developed during the BebeR-initiative (Pundt and Heilmann 2020; Pundt 2018). It resulted finally in an interactive, online mapping system including a great variety of spatial datasets (layers) from different sectors. To implement the system, standardized Geographical Information Services were used. The aim was to enable users to overlay different spatial data layers aiming at the analysis of data from different perspectives and thus broadening the information basis for decision making. In such a way, the concept of the online mapping system follows the "Open by Default"-approach, as described in figure 1. The integration of spatial data from different sources was appreciated very much by the project partners because before starting the project most actors had only access to their "own" data, which implied a relatively "closed view" ("blinkers") on the problems that occur due to climate change. Using the data from the online mapping system, it was possible, for example, to integrate data in such a way that areas vulnerable to soil erosion were identified under consideration of water-, soil-, geological-, agricultural-, vegetation-related, infrastructural-, and other information. The growth of the database increased the opportunities for participants to look on the different data layers in a kind of a "holistic" 
information platform. The system enabled users to integrate datasets aimed at more sophisticated analyses. This led to new maps, which, in turn, could be provided for other users via the same system. In such a way, the online mapping system was soon accepted as an ideal basis for discussion of problem solutions, and alternative approaches, considering not only a "sector-wise", but varying perspectives and scenarios. The maps became decision support tools on the one hand, and information means for the actors, as well as the public.

However, in some cases, it was not easy to convince administrational officers to provide their datasets as "open" layers in the mapping system due to legal and security reasons. Apart from such official datasets, openly accessible spatial data was integrated, e. g. topographical data from the world-wide OpenStreetMap project, "a map of the world, created by people like you and free to use under an open license" (OSM 2021), and data from citizen-based mapping initiatives to collect information on invasive species (KORINA 2021; see Figure 2).

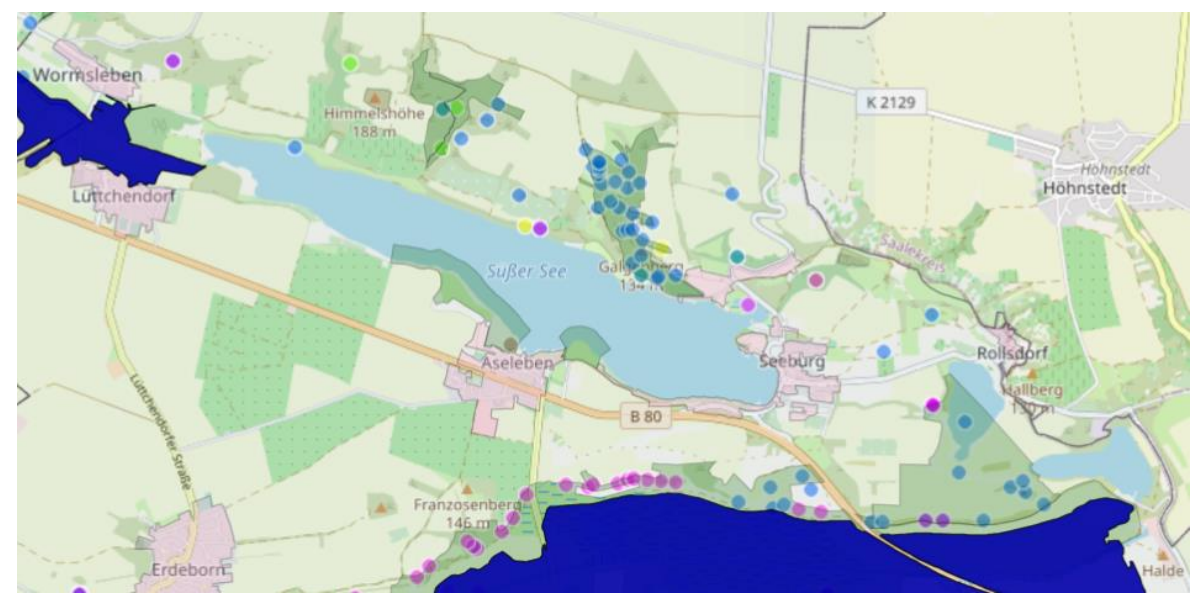

Figure 2. Official, administrative data (dark area objects: flooding areas), as well as open, spatial data (point objects: invasive species and underlying topography: OpenStreetMap) in one map. Both kinds of data are integrated and used by different stakeholders to support decision making in spatial planning

\subsection{Special Considerations Concerning Decision Making}

During workshops, the maps and different alternatives of measures to reduce soil erosion in view of climate chnge were discussed intensively within the framework of the BebeR-project. Selected measures to reduce soil erosion in vulnerable areas were prioritized and those considered as being the "most important and feasible" measures were selected to be realized within the next few months or years in the pilot region. The following list mentions some of the discussed options:

- Minimization of soil erosion through change of agricultural practices

- $\quad$ Storage of great water amounts occurring during heavy rainfall events

- Reduction of flow velocity in rivers to reduce erosion potential

- Analyzing habitats of invasive plants based on various spatial datasets; developing concepts to mitigate invasive species in certain areas, followed by afforestation or reafforestation aimed at reducing soil erosion

The online mapping system served as a comprehensive and reliable data basis to analyze data and compose maps that were used as basis for discussion, and (visual) decision support. Different stakeholders were applying the system for a variety of purposes, but foremost as a means to understand the different perspectives and opportunities, thus coming finally to common results.

\section{CONCLUSIONS}

Based on an "Open by Default" - approach, the BebeR-online mapping system provides both, open spatial data, as well as official administrative data and data from other providers. Open spatial data result from well-known initiatives as OpenStreetMap (OSM), the "fastest growing community mapping project of all 
times" (Digital Geography 2021), as well as data provided from citizen-science (or VGI-) initiatives (KORINA 2021). When implementing the system, the main difficulties occurred not technically. Due to the usage of standardized Geographic Information Services, the technical problems were predominantly solved. However, the discussions with stakeholders to convince them to provide the data in an open, commonly accessible web-based system were quite time consuming in some cases. Even the arguments that good decisions on spatial problems require the availability of at least all data that concern the problem to be solved, was sometimes not enough to persuade representatives. Therefore, the online mapping system, originally planned as "completely open”, was secured in a way that users only could access data when registering as a legal user, by Email address and Password. Jackson et al (2013) hint on differences between web-based spatial datasets concerning accuracy and completeness, and that the "vagueness can be degradation in the utility of VGI for decision making; however, these effects have not been studied" (Jackson et al 2013). This deficit does probably not only affect positional accuracy and completeness, but more, e. g. semantic accuracy, granularity of attributes describing spatial objects, and reliability, as well as data security aspects. This needs further research in future.

Finally, the users of the BebeR-system were well-disposed to the application because it contains both, official, administrative data, and open spatial data. But it must be added with emphasis, that open data are only accepted as information basis, if specific qualitative requirements are fulfilled. Clearly, the stakeholders participating in BebeR uttered that they would prefer to renounce on the usage of open data, if such quality parameters are not ensured. Especially concerning administrative decision-making, open spatial data can hardly be included in analyses and resulting maps, and therefore in decision-making, if they do not serve the corresponding legal, and qualitative requirements.

\section{REFERENCES}

Digital Geography, 2021, How to use OpenStreetMap, guides for beginners and advanced users. https://digitalgeography.com/use-openstreetmap-guides-beginners-advanced-users-learnosm-org/ (accessed 09-01-2021)

Goodchild, M., 2007. Citizens as Sensors: The World of Volunteered Geography. GeoJournal 69(4), pp 211-221.

Horster, E., Kärle, E., 2019. Braucht der Tourismus Open Data? Open Data Germany. https://open-datagermany.org/braucht-der-tourismus-open-data/ (accessed 19-01-2021)

Jackson, S.P., Mullen, W., Agouris, P., Crooks, A., Croitoru, A., Stefanidis, A., 2013. Assessing Completeness and Spatial Error of Features in Volunteered Geographic Information. ISPRS Int. Journal of Geo-Information 2, pp. 507-530.

Kitchin, R., 2014. The Data Revolution. Big Data, Open Data, Data Infrastructures and their Consequences. SAGE Publications, London.

Klessmann, J., Denker, P., Schieferdecker, I., Eschulz, S.E., 2012. Open Government Data Deutschland.

KORINA, 2021. Koordinationsstelle Invasive Neophyten in Schutzgebieten Sachsen-Anhalts. https://www.korina.info/ (accessed 05-01-2021)

Kucera, J., Chlapek, D., 2014. Benefits and Risks of Open Government Data. Journal of Systems Integration 2014/1, pp. 30-41.

NibusinessInfo, 2021. Open data opportunities for business. https://www.nibusinessinfo.co.uk/content/open-dataopportunities-business (accessed 01-11-2020)

OKF, 2021. Open Knowledge Foundation - The Open Data Handbook. Available from: https://opendatahandbook.org/guide/en/ (accessed 15-01-2021)

OSM (2021): OpenStreetMap. https://www.openstreetmap.org/ (accessed 20-01-2021)

Pundt, H., Heilmann, A., 2020. Building Collaborative Partnerships: An Example of a 3rd Mission Activity in the Field of Local Climate Change Adaptation. In: Leal, W.F., Alves, F., Azeiteiro, U., Manolas, E.: Universities as Living Labs for Sustainable Development. Supporting the Implementation of the Sustainable Development Goals: Vol. II., Springer Nature Switzerland, Cham, pp 621-636.

Pundt, H., 2018. Collaboration of Science, Practice and Citizens in the Field of Climate Change Adaptation, supported by Interactive GI-Tools. 21st Int. Conference on Geographic Information Sciences Lund/Sweden. Proceedings, https://agile-online.org/programme-2018/accepted-papers-and-posters-2018.

Van Loenen, B., Vancauwenberghe, G., Croempvoets, J., 2018. Open Data Exposed. Information Technology and Law Series, Vol. 30. T.M.C. Asser Press, Springer. 